\title{
Long-Term Outcomes Following Surgical Management of Urethral Catheter Injuries in Men With Spinal Cord Injury
}

\author{
Kirtishri Mishra, ${ }^{1,2}$ Rodrigo A. Campos, ${ }^{3}$ Laura Bukavina, $, 1,2$ Reynaldo G. Gómez ${ }^{\bowtie 3}$ \\ ${ }^{1}$ Urology Institute, University Hospitals Cleveland Medical Center, Cleveland, United States ${ }^{2}$ Case Western Reserve University School of Medicine, Cleveland, \\ United States ${ }^{3}$ Urology Service, Hospital del Trabajador, Santiago, Chile
}

\begin{abstract}
Introduction To evaluate the outcomes of surgical management of men with spina cord injury (SCI) with subsequent catheter damage to the urethra that prevents clean intermittent catheterization (CIC).

Materials and Methods We performed a retrospective analysis of male SCI individuals on CIC with catheterinduced urethral injuries who had undergone an operative intervention in the last 30 years at our institution. The offered surgical managements were direct vision internal urethrotomy (DVIU) or urethroplasty (UP). Continent diversion (CDIV) was indicated when reconstruction was not possible.
\end{abstract}

Results A total of 43 male SCI patients were identified. Median age was 50 years (IQR 41 to 57), and follow-up was 69 months (IQR 34 to 125). Inability to perform CIC was due to urethral stricture (25), false passages (11), fistula (4), diverticulum (2), and urethral erosion (1). Primary intervention techniques were DVIU, UP, and CDIV. Overall primary success, defined by the ability to return to continent CIC, was 25/43 (58\%); secondary surgery (10 CDIV, 3 UP, 1 DVIU) rescued 14/18 failures for a final 91\% success rate.

Conclusion Urethral injuries in men with SCI are complex, but individualized continued surgical management can be successful in up to $90 \%$ of patients. Therefore, reconstruction should be considered in this population to restore continent intermittent catheterization.

\section{Introduction}

Urologic care in individuals with a history of spinal cord injury (SCI) continues to pose a medical challenge for urologists[1-5].There are advantages and disadvantages to each approach the provision of urologic care for a patient with a neurogenic bladder. Since its introduction in the early 1970s by Lapides and Diokno, clean intermittent catheterization (CIC) has become the mainstay for the management of neurogenic bladder in the SCI population [6,7]. Before the routine use of $\mathrm{CIC}$, the leading cause of death in these patients was renal failure and urinary sepsis $[2,8,9]$. Those patients with an open sphincter/external sphincterotomy were managed with a condom catheter, and those with a closed sphincter were subjected to a chronic indwelling catheter, with urinary diversion being the most invasive option[1].

\section{Key Words}

Catheter urethral injury, urethral reconstruction, urethroplasty, intermittent catheterization, neurogenic bladder, spinal cord injury, reconstructive urology

\section{Competing Interests}

None declared

\section{Article Information}

Received on January 11, 2021 Accepted on April 3, 2021

Soc Int Urol J.2021;2(3):144-150

DOI 10.48083//AGBN5610 


\section{Abbreviations}

CDIV continent diversion

CIC clean intermittent catheterization

DBMG dorsal buccal mucosa graft urethroplasty

DVIU direct vision internal urethrotomy

SCI spinal cord injury

SPT suprapubic tube

UP urethroplasty

While condom catheters, indwelling catheters, and CIC are all efficacious in appropriately selected patients, they each have their own shortcomings[10]. A condom catheter can lead to a diverticulum, stricture, or erosion, a chronic indwelling catheter may lead to erosion and recurrent urinary tract infections, and CIC can lead to a false passage, stricture, or a fistula. In fact, numerous studies indicate that with a long enough course of chronic instrumentation and repeated trauma, most of these patients suffer some degree of catheter related complication[10-12].

In our practice at a large volume SCI rehabilitation referral center, we strongly advocate for maintaining urinary continence by means of CIC, instead of incontinent condom catheter or chronic indwelling catheters. However, as may be expected, over time the urethra is at risk for damage and complications[12]. Within the reconstructive field, there is continued debate about the utility of reconstruction in these patients, with some providers arguing that there is a high likelihood of failure $[13,14]$. Therefore, incontinent urinary diversions, such as an ileal conduit or an indwelling catheter, are used instead of reconstructive options. However, we believe that there is a benefit in restoring continent CIC, minimizing the use of indwelling catheters or external appliances, particularly for individuals with SCI who are already self-conscious about their appearance.

The goal of this study was to evaluate our experience of more than 30 years to determine whether interventions to restore continent CIC in these patients provides a durable option for urinary management. We hypothesize that catheter-induced urethral complications in SCI patients can be successfully managed with a selective surgical approach that can restore continent CIC and that should be routinely considered in this population.

\section{Materials and Methods}

With the approval of the Institutional Review Board (CEC/12/2020), we undertook a retrospective review of SCI patients with surgically managed with DVIU, UP, or CDIV and subsequent catheter urethral injuries from March 1988 to December 2019. Additional patient inclusion criteria were age $>18$ years and complete medical records and $>10$ months of follow-up. The procedures were performed by 6 different surgeons with half of the cases being performed by RGG.

Our institution is a high-volume tertiary referral center for trauma and rehabilitation, specializing in work-related injuries. After trauma stabilization, SCI patients are admitted to a comprehensive rehabilitation program that includes regular annual visits for life after discharge. Urological management is based on CIC, and able patients are quickly instructed in selfcatheterization. Baseline urodynamics and upper tract imaging are performed at the end of the spinal shock; afterwards, patients are followed with lifelong regular visits as required. Treatment is oriented to obtain a good capacity and low-pressure continent bladder. Anticholinergics, botulinum toxin, bladder augmentation, or sphincter reinforcing procedures are indicated as needed. Long-term periodic monitoring includes renal function test and urinary tract imaging.

Individuals presenting with catheter urethral injuries making CIC difficult or impossible were evaluated with endoscopy and urethrography. All patients considered as good surgical candidates were offered surgery. Surgical management was individualized according to the type of urethral pathology, including direct vision internal urethrotomy (DVIU), urethroplasty (UP), or continent diversion (CDIV). DVIU was used in patients with bulbar strictures up to $2 \mathrm{~cm}$ in length. Penile strictures, bulbar strictures $>2 \mathrm{~cm}$, or any recurrence after DVIU were managed with a dorsal buccal mucosa graft (DBMG) urethroplasty. Posterior (membranous) urethral strictures were mostly related to neurogenic spasticity of the external sphincter and were managed with a DVIU plus simultaneous intrasphincter botulinum toxin injection. Diverticula were managed with tailoring and closure, with use of DBMG if a stricture was present. Penile urethral erosions were managed with primary closure with or without DBMG, depending on urethral plate. Fistulas were managed with resection and repair of concomitant stricture, diverticulum, or false passage, with use of DBMG as necessary. Continent diversion (CDIV) was indicated after failure of other options or when reconstruction was not possible. Our preference is for a continent cutaneous ileal cecocystoplasty as it avoids ureteral reimplantation, provides good bladder augmentation, and provides a reliable continent channel for catheterization.

An indwelling urethral silicone catheter is left for 2 to 3 days after DVIU and for 3 weeks after UP, before CIC is resumed. Pericatheter urethrography is performed before catheter withdrawal. Although reusable polyurethane catheters are the standard, hydrophilic coated catheters are prescribed after urethral surgery. 
CDIV patients are left with a catheter through the channel and a discharge suprapubic tube (SPT) cystostomy for 3 weeks, and CIC is started through the channel afterwards.

A continued surgical management protocol was followed; therefore, patients who failed primary intervention were evaluated and offered individualized secondary rescue surgery. Those who were not candidates for further surgery were managed with a definitive SPT. False passages managed by simple urethral catheter stenting and external sphincter spasticity receiving botulinum toxin without associated urethral stenosis were not included in this series.

The goal of all procedures was to recover easy, non-traumatic, continent CIC. Failure was defined as inability to return to continent CIC requiring a secondary surgical procedure or definitive SPT. The Clavien-Dindo classification was used for surgical complications.

\section{Results}

There was a total of 43 patients, with a median age of 50 years (IQR 41 to 57 ) and follow-up of 69 months (IQR 34 to 125). All patients were compliant with their regular appointments and no patient was lost to follow-up. Five individuals died from unrelated causes 23 to 293 months after the initial surgery.

Figure 1 summarizes the urethral pathology. Four patients with false passage and both patients with diverticulum also had a stricture; these patients are not counted doubly as strictures.

Table 1 describes the types of injuries and the primary surgical interventions with associated success

\section{FIGURE 1.}

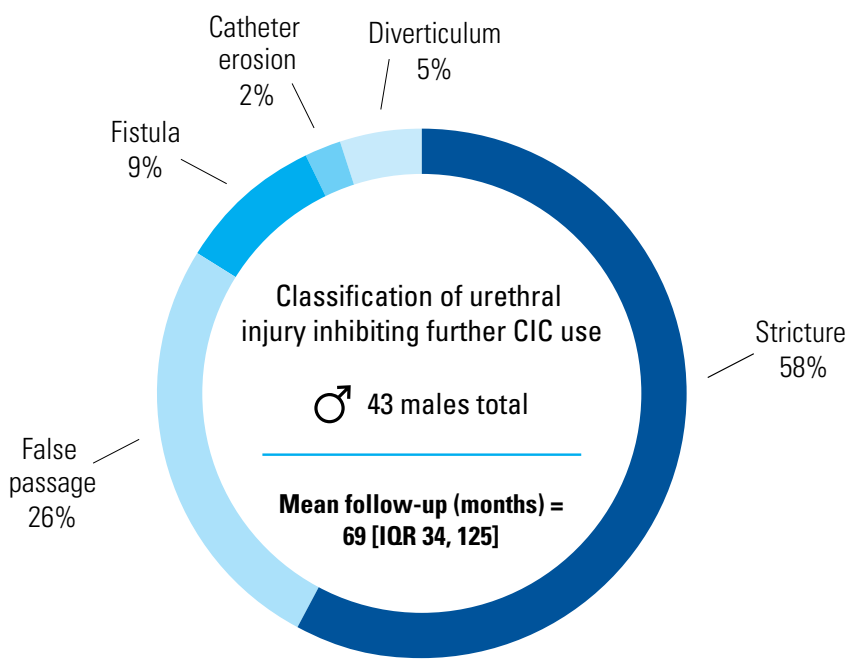

Type of urethral injury

\section{TABLE 1.}

The cohort categorized by the presenting pathology and the associated interventions. Success was defined as the ability to return to continent intermittent catheterization

\begin{tabular}{|c|c|c|}
\hline Type of Injury & $\begin{array}{c}\text { Primary procedure } \\
\text { (patients) }\end{array}$ & $\begin{array}{c}\text { Success } \\
\text { n }(\%)\end{array}$ \\
\hline \multirow{3}{*}{ Stricture } & DVIU (16) & $9(58)$ \\
\hline & UP (6) & $5(83)$ \\
\hline & CDIV (3) & $2(67)$ \\
\hline Total & 25 & $58(64)$ \\
\hline \multirow{3}{*}{ False passages } & UP (4) & $2(50)$ \\
\hline & CDIV (6) & $3(50)$ \\
\hline & DVIU (1) & $0(0)$ \\
\hline Total & 11 & $5(45)$ \\
\hline \multirow{2}{*}{ Fistulas } & $U P(2)$ & $0(0)$ \\
\hline & CDIV (2) & $2(100)$ \\
\hline Total & 4 & $2(50)$ \\
\hline Catheter erosion & UP (1) & $0(0)$ \\
\hline Total & 1 & $0(0)$ \\
\hline Diverticulum & UP (2) & $2(100)$ \\
\hline Total & 2 & $2(100)$ \\
\hline Overall & 43 & $25(58)$ \\
\hline
\end{tabular}

rates. The primary success rate for the cohort as a whole was calculated at $25 / 43(58 \%)$.

Table 2 highlights the outcomes according to the type of surgical intervention. The primary success of DVIU, UP, or CDIV was fairly similar $(53 \%, 64 \%$, and $58 \%$, respectively); however, CDIV had the highest perioperative morbidity (41\%), with $36 \%$ of the patients suffering Clavien-Dindo $\geq 3$ complications. In contrast, DVIU and UP had $17 \%$ and $18 \%$ morbidity, with none of the patients in the DVIU group suffering a ClavienDindo $\geq 3$ complication. Complications are listed in Table 3. Complications were observed in 15/57 (26\%) of procedures, and 10 of them (18\%) were Clavien-Dindo $\geq 3$. The most common complication was chimney failure (5), which refers to anything that leads to channel kinking and difficult catheter passage needing revision. 
The continued management of primary failures is also shown in Table 2. Rescue surgery was offered to 14 of 18 patients in whom primary failure occurred, while the remaining 4 patients were not considered surgical candidates and were left with a SPT. All secondary procedures were successful in re-establishing CIC. The final success of DVIU was 10/18 (56\%), of UP 12/17 (71\%), and CDIV $17 / 22$ (77\%). Of the 10 patients in the DVIU and botulinum toxin group, 6 did not require any further procedure, while the remaining 4 patients required UP (2), CDIV (1), and SPT (1). Additionally, the re-do CDIV (Table 2) group refers to failure of the channel requiring a completely new channel. Isolated stomal stenosis was reconstructed at the stomal level only. Ultimately, after a total of 57 surgical procedures, the reconstruction goals were achieved in 39 of 43 patients (a $91 \%$ overall success rate), and there was no surgical mortality.

\section{Discussion}

The management of neurogenic bladder in patients with SCI continues to be an evolving issue [1-5]. Since the advent of CIC, most providers would agree that patients with good upper extremity function status should be afforded this modality of bladder management. Although recently reported patient outcomes suggest individuals with SCI may prefer indwelling catheters for convenience, there is support in the literature for the benefits of avoiding long-term indwelling catheters or external condom catheters, as well as for the benefits of avoiding major surgeries for urinary diversion $[6,7,15,16]$. Because of this, we believe every reasonable effort should be made to maintain CIC.

There is a lack of consensus on the optimal management strategy for patients who are unable to continue performing CIC because of catheter-induced urethral pathology, such as obstruction, false passage, diverticulum, or fistula, and very little has been published on this issue. In this study, we investigated the outcomes of our management of these described pathologies over the course of 30 years at our institution [13]. We hypothesized that surgical interventions aimed at restoring continent CIC would be successful in the majority of these patients.

Overall, we found that $91 \%$ of patients who underwent continued surgical intervention to restore CIC were successful through the course of our followup. These interventions included DVIU, UP, and CDIV. The secondary procedure was determined on the basis of the status of the urethra. DVIU is considered as the first option, mainly for short and simple strictures; for longer, heavy fibrous or complex cases (like associated false passages) a more definitive reconstruction was necessary. Unreconstructible urethras were considered for diversion. Although DVIU showed the lowest success

\section{TABLE 2.}

The cohort categorized by the type of surgical intervention performed. The overall final success rate was $91 \%$ and only 4 patients ultimately failed surgical interventions and required a definitive suprapubic tube

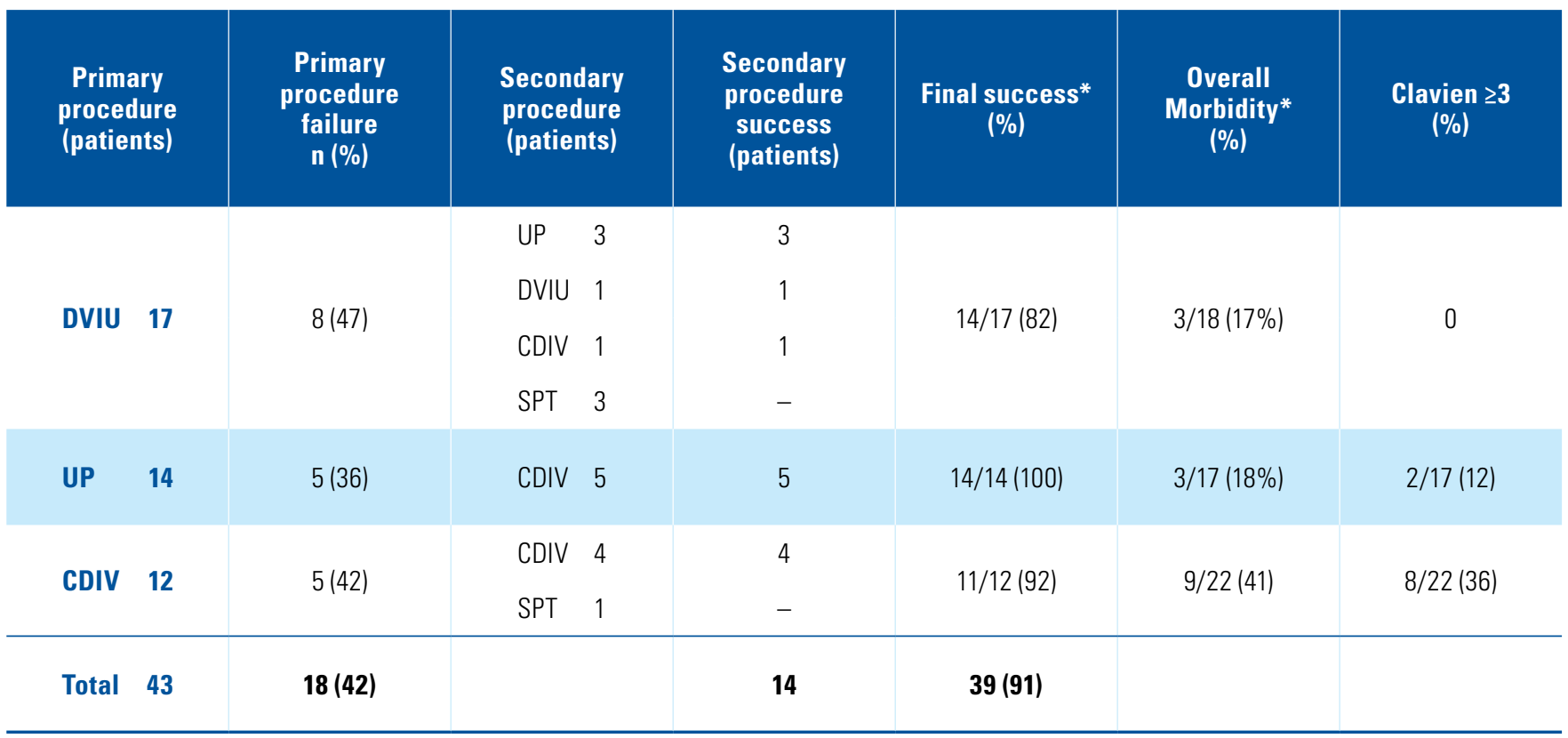

* Final success and overall morbidity were calculated combining primary and secondary surgeries. 
(56\%), as mentioned above it is the best choice for short bulbar and membranous strictures because of its simplicity and low complication rate. In contrast, CDIV achieved the highest success (77\%) but was linked to the highest morbidity.

The UP subgroup had a $71 \%$ success rate, which contrasts with previous reports. Specifically, in 2003, Secrest et al. noted a $65 \%$ (11/17 patients) failure rate in urethral reconstruction in a similar cohort, so the authors concluded that these patients are best treated with urinary diversion from the beginning[13]. In our series, however, we found that $71 \%$ of the patients who underwent a UP were able to successfully return to $\mathrm{CIC}$, avoiding a diversion and with a tolerable $18 \%$ complication rate.

Our results also emphasize the value of continued surgical management: 14 of the 18 failures (and 33\% of the whole series) were taken for selective individualized secondary surgery with $100 \%$ secondary success. Reconstruction was considered a failure in only 4 patients (9\%), who left with a chronic SPT. After these results, providers should strongly consider reconstruction for patients who are good candidates to restore continent CIC.

The field of reconstruction has evolved significantly in the last 15 years, so that instead of reported $65 \%$ failure rate, most studies now cite a $70 \%$ to $90 \%$ success rate after urethroplasty[17]. Urethral reconstruction is an evolving art, with more fellowship-trained reconstructive urologists practicing at the current time, with an improvement in graft techniques and postoperative care[18]. In 2004, Ronzoni et al. cited a $73 \%$ success rate in 48 neurological patients with urethral diverticulum[19]. Following this finding, Meeks et al. reported a $63 \%$ success rate after urethroplasty to restore CIC in 2008[20]. They cited the presence of a good urethral plate as an important prerequisite for successful repair. In a follow-up study by the same group, Casey et al. reported a $70 \%$ success rate in 23 neurogenic patients after urethral reconstruction, with no patients requiring urinary diversion[21]. Of note, we favor a dorsal buccal mucosal graft urethroplasty as we believe it allows a smoother passage of the catheter for patients performing CIC; a ventral graft or flap may produce ventral irregularity, which may be a risk factor for a false passage.

It is important to acknowledge that despite improvement in techniques and postoperative management, reconstruction failure remains a possibility, and the surgeon must have a secondary procedure in mind for these patients. Therefore, patients should be thoroughly evaluated before undergoing procedures, and should be provided comprehensive counseling to ensure they are aware that they have a
TABLE 3.

List of observed complications

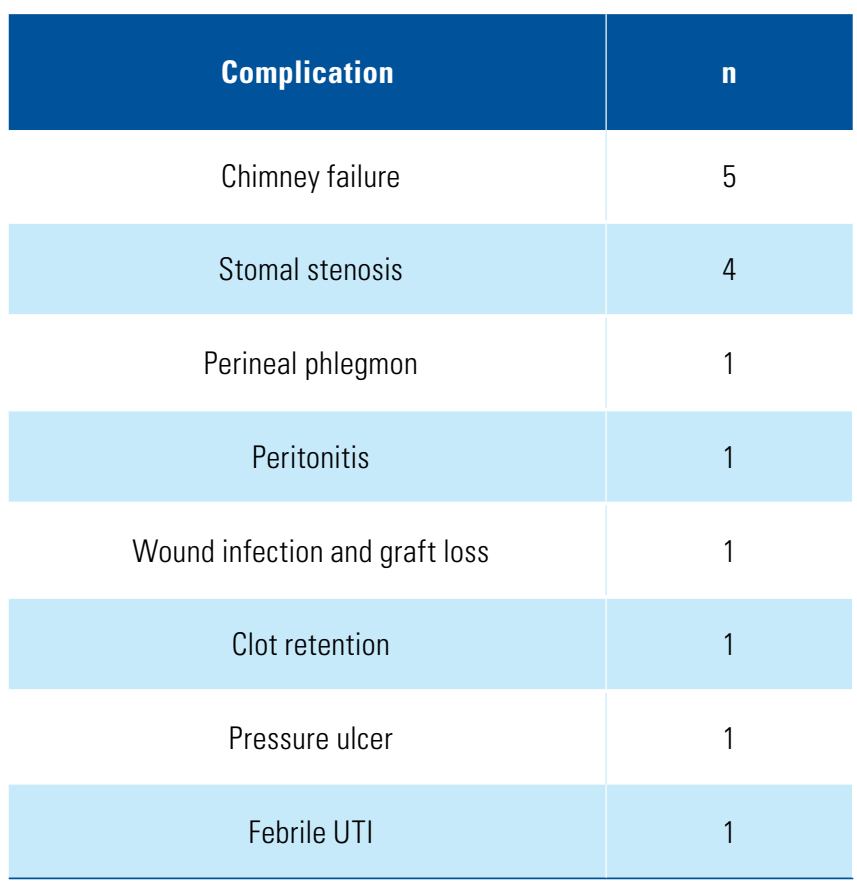

higher failure rate than non-neurogenic patients. Factors that may lead to worse outcomes include but are not limited to poor tissue quality, poor nutrition, subpar hygiene, lack of social support, and persistent insults to the urethra. Strict postoperative care with delayed wheelchair usage and avoidance of perineal pressure may prevent wound breakdown and pressure ulcers. Furthermore, the outcome is also associated with the complexity of the case and team's experience. If any of these issues are non-modifiable and appear to be a significant impediment to undertaking a reconstruction, then diversion should be considered.

Factors that may stratify a urethra as "unreconstructible" include persistent or recurrent fibrosis, large false passage with persistent infection, large fistula, severe panurethral stricture, poor vascularity of the urethral plate, poor quality of local tissues, and a small capacity bladder requiring bladder augmentation. In these patients, a continent or incontinent urinary diversion with or without bladder sparing should be considered. The authors of this study favor bladdersparing continent diversion if possible. The advantages of such diversion are that no ureteral reimplantation is needed and patients can continue to perform continent CIC via a catheterizable channel (continent cutaneous ileal cecocystoplasty, or channels like Mitrofanoff, Yang-Monti, or Casale). Bladder augmentation may be considered in these patients if appropriate. Patients with poor performance status or recurrent reconstruction failures are elected for a definitive suprapubic tube placement. 
One of the weaknesses of this study is its retrospective nature. However, most of these patients were treated by a single provider (RGG), who operated on and managed the follow-up care of these patients for most of the 30-year follow-up. Furthermore, despite being a comparatively large series with SCI individuals who underwent reconstructive urethral surgery, this study still has a limited cohort. Overall, this shortcoming can be addressed with a prospective analysis, which may require a multi-institutional effort.

\section{Conclusion}

We present our long-term results of surgical reconstructive management of catheter-induced urethral injuries in the male SCI patient. A selective surgical approach is described, depending on the type of injury and patient's condition. Our findings suggest that surgery should be considered to restore continent catheterization. While the primary success rate is lower than in non-SCI cases, properly selected secondary rescue surgery may improve success up to $90 \%$, so reconstruction is well worth the effort to restore continent CIC in these complex cases.

\section{Author Contributions}

Kirtishri Mishra: data analysis, manuscript writing and editing; Laura Bukavina: data analysis, figure, manuscript writing and editing; Reynaldo G. Gómez: protocol and project management, data analysis, manuscript writing and editing; Rodrigo A. Campos: data collection. 


\section{Reference}

1. Larsen LD, Chamberlin DA, Khonsari F, Ahlering TE. Retrospective analysis of urologic complications in male patients with spinal cord injury managed with and without indwelling urinary catheters. Urology.1997;50(3):418-422. doi: 10.1016/S0090-4295(97)00224-0.

2. Weld KJ, Dmochowski RR. Effect of bladder management on urological complications in spinal cord injured patients. J Urol.2000;163(3):768-772.

3. Singh R, Rohilla RK, Sangwan K, Siwach R, Magu NK, Sangwan SS. Bladder management methods and urological complications in spinal cord injury patients. Indian J Orthop.2011;45(2):141-147. doi: 10.4103/0019-5413.77134.

4. Anderson CE, Chamberlain JD, Jordan X, Kessler TM, Luca E, Möhr S, et al. Bladder emptying method is the primary determinant of urinary tract infections in patients with spinal cord injury: results from a prospective rehabilitation cohort study. BJU Int.2019;123(2):342-352. doi: 10.1111/bju.14514. Epub 2018 Sep 9.

5. Kiddoo D, Sawatzky B, Bascu C-D, Dharamsi N, Afshar K, Moore KN. Randomized crossover trial of single use hydrophilic coated vs multiple use polyvinylchloride catheters for intermittent catheterization to determine incidence of urinary infection. J Urol.2015;194(1):174-179. doi: 10.1016/j.juro.2014.12.096. Epub 2015 Jan 10.

6. Lapides J, Diokno AC, Silber SJ, Lowe BS. Clean, intermittent self-catheterization in the treatment of urinary tract disease. J Urol.1972;107(3):458-461. doi: 10.1016/s0022-5347(17)61055-3.

7. Bloom DA. The retrograde idea of Jack Lapides: clean intermittent catheterization. J Urol.2017; 197(2S):S125-S126. doi: 10.1016/j. juro.2016.10.080. Epub 2016 Dec 20

8. Lapides J, Diokno AC, Silber SJ, Lowe BS. Clean, intermittent self-catheterization in the treatment of urinary tract disease. J Urol.2017;197(2S):S122-S124. doi: 10.1016/j.juro.2016.10.097. Epub 2016 Dec 21.

9. Perrouin-Verbe B, Labat JJ, Richard I, Mauduyt de la Greve I, Buzelin JM, Mathe JF. Clean intermittent catheterisation from the acute period in spinal cord injury patients. Long term evaluation of urethral and genital tolerance. Paraplegia.1995;33(11):619-624. doi: 10.1038/ sc.1995.131.

10. Chai T, Chung AK, Belville WD, Faerber GJ. Compliance and complications of clean intermittent catheterization in the spinal cord injured patient. Paraplegia.1995;33(3):161-163. doi: 10.1038/ sc.1995.35

11. Afsar SI, Yemisci OU, Cosar SNS, Cetin N. Compliance with clean intermittent catheterization in spinal cord injury patients: a longterm follow-up study. Spinal Cord.2013;51(8):645-649. doi: 10.1038/ sc.2013.46. Epub 2013 Jun 11.
12. Krebs J, Wollner J, Pannek J. Bladder management in individuals with chronic neurogenic lower urinary tract dysfunction. Spinal Cord.2016;54(8):609-613. doi: 10.1038/sc.2015.196. Epub 2015 Nov 10.

13. 1Secrest CL, Madjar S, Sharma AK, Covington-Nichols C. Urethral reconstruction in spinal cord injury patients. J Urol.2003;170/4 Pt 1):1217-1221; discussion 1221. doi: 10.1097/01.ju.0000087614.19425.df.

14. Stoffel JT, McGuire EJ. Outcome of urethral closure in patients with neurologic impairment and complete urethral destruction. Neurourol Urodyn.2006;25(1):19-22. doi: 10.1002/nau.20146.

15. Cornejo-Davila V, Duran-Ortiz S, Pacheco-Gahbler C. Incidence of urethral stricture in patients with spinal cord injury treated with clean intermittent self-catheterization. Urology.2017;99:260-264. doi: 10.1016/j.urology.2016.08.024. Epub 2016 Aug 23.

16. Myers JB, Lenherr SM, Stoffel JT, Elliott SP, Presson AP, Zhang C, et al. Patient reported bladder related symptoms and quality of life after spinal cord injury with different bladder management strategies. J Urol.2019;202(3):574-584. doi: 10.1097/JU.0000000000000270. Epub 2019 Aug 8.

17. Soave A, Kluth L, Dahlem R, Rohwer A, Rink M, Reiss P, et al. Outcome of buccal mucosa graft urethroplasty: a detailed analysis of success, morbidity and quality of life in a contemporary patient cohort at a referral center. BMC Urol.2019;19(1):18. doi: 10.1186/ s12894-019-0449-5

18. Saavedra AA, Rourke KF. Training in reconstructive urology: the past, present and future. Trans/ Androl Urol.2018;7(4):666-672. doi: 10.21037/tau.2018.03.04

19. Ronzoni G, De Giovanni L, Manca A, Pasqui F, Mastrangelo P, Menchinelli P. Urethroplasty in recurrent urethral diverticula in neurological patients: long-term results using a personal technique. BJU Int.2004;94(1):185-187. doi.org/10.1111/j.1464-410X.2004.04917.x

20. Meeks JJ, Erickson BA, Helfand BT, Gonzalez CM. Reconstruction of urethral erosion in men with a neurogenic bladder. BJU Int.2009;103(3):378-381. doi: 10.1111/j.1464-410X.2008.08020.x. Epub 2008 Sep 3.

21. Casey JT, Erickson BA, Navai N, Zhao LC, Meeks JJ, Gonzalez CM. Urethral reconstruction in patients with neurogenic bladder dysfunction. J Urol.2008;180(1):197-200. doi: 10.1016/j. juro.2008.03.056. Epub 2008 May 21. 